\title{
Transition from Symmetric to Asymmetric Scaling Function before Drop Pinch-Off
}

\author{
Alexander Rothert, Reinhard Richter, and Ingo Rehberg \\ Physikalisches Institut, Experimentalphysik V, Universität Bayreuth, D-95440 Bayreuth, Germany
}

(Received 21 December 2000; published 6 August 2001)

\begin{abstract}
The drop pinch-off at a nozzle is studied experimentally for a glycerin-water mixture in surrounding air. The neck diameter of the fluid shrinks with constant velocity. After a distinct transition point, the shrink velocity switches to a smaller value. Before that transition point, the shape of the neck can well be described by a symmetric scaling function, as obtained from Stokes-flow theory of drop formation. This function gives way to an asymmetric scaling function in the final stage before pinch-off.
\end{abstract}

DOI: $10.1103 /$ PhysRevLett.87.084501

The phenomenon of drop formation had been investigated by Mariotte as early as 1718 [1]. Since then, the first stage of the instability has attracted most of the interest, because it is accessible by classical linear stability analysis [2]. In the past couple of years, however, attention has switched to the flow regime in the immediate vicinity of the pinch-off of the tiny liquid thread connecting two neighboring drops. This is mainly due to the practical implications an understanding of this final stage of drop formation can have to a lot of technical applications such as spraying and ink-jet printing. A recent review has been given by Eggers [3].

The first theoretical analysis of the physics in the pinch-off region was conducted by Lee [4]. Starting from the Navier-Stokes equation, he gave a one-dimensional description of the drop shape and the velocity field for inviscid liquids. Eggers [5] considered the same for the case of a viscous fluid with the additional ansatz of self-similarity. As a result, he obtained universal scaling functions $\Phi(\xi)$ and $\Psi(\xi)$ describing the drop profile and the velocity field inside the neck for a Navier-Stokes flow just before the pinch-off. According to Eggers [5] the drop profile, i.e., the radius $h$ of the thread as a function of position $z$ and time $t$, is given by

$$
h(z, t)=l_{\nu}\left|t^{\prime}\right| \Phi(\xi) .
$$

Here $t^{\prime}=\left(t_{0}-t\right) t_{\nu}^{-1}$ is the scaled time distance to the pinch-off taking place at $t=t_{0}$ and $l_{\nu}=\nu^{2} \varrho \sigma^{-1}$ and $t_{\nu}=\nu^{3} \varrho^{2} \sigma^{-2}$ are an intrinsic length and time where $\nu$, $\varrho$, and $\sigma$ denote the kinematic viscosity, the density, and the surface tension of the fluid, respectively. The similarity variable is written by $\xi=\left(z-z_{0}\right) l_{\nu}^{-1}\left|t^{\prime}\right|^{-\beta}$. Taking into account the full Navier-Stokes equations, Eggers obtained scaling functions which are asymmetric with respect to the pinch-point $z_{0}$, and a scaling exponent of $\beta=0.5$ [5]. A comparison of that theory with experimental data was presented by Kowalewski [6]. In the last stage before pinch-off, the similarity solution $\Phi$ becomes unstable against the formation of satellite drops [7,3]. Brenner et al. [8] have found numerically an infinite series of competing higher order solutions. These solutions have not yet been observed.
PACS numbers: 47.55.Dz, 47.20.Dr

A different set of scaling functions was derived by $\mathrm{Pa}-$ pageorgiou for the case of a viscosity dominated flow [9]. For initially small Reynolds numbers and far away from the pinch point, the inertial terms can be neglected and the flow is governed by the Stokes equation. Here, the scaling functions are symmetric with respect to $z_{0}$ and the argument $\xi=\bar{\xi}\left(z-z_{0}\right) l_{\nu}^{-1}\left|t^{\prime}\right|^{-\beta}$ is determined only up to an arbitrary normalization length $\bar{\xi}[9,3]$. Because the exponent $\beta$ is close to 0.175 , the axial scale $z$ contracts at a much slower rate than it does for the case of Eggers' solution. However, with decreasing time distance $t^{\prime}$ to the pinch point and increasing axial velocity, the assumption of negligible inertia is no longer satisfied. Thus the symmetric solution must finally give way to the asymmetric one. When and how this takes place is unresolved in experiment and theory [3].

In the present paper, we demonstrate this transition experimentally. The profiles of drops of a glycerin-water mixture falling in air are analyzed quantitatively utilizing the symmetric and asymmetric scaling functions. For the temporal evolution of the neck radius we obtain the two predicted scaling regimes, separated by a distinct transition point.

The experimental setup is shown in Fig. 1. A syringe pump serves for a constant flow rate of $0.5 \mathrm{ml} \mathrm{min}^{-1}$. The syringe is connected via a Verdoprene flexible tube to a Hirschmann pipette tip at whose lower part the drop formation occurs. The outer diameter of the nozzle wetted by the fluid amounts to $1 \mathrm{~mm}$ at the orifice. Background illumination is provided with a cold light source diffused by a frosted glass. A high-speed-CCD camera (Kodak Ektapro High-Spec Motion Analyzer) detects the falling drops with $239 \times 192$ pixels of $32 \times 32 \mu \mathrm{m}$ at record

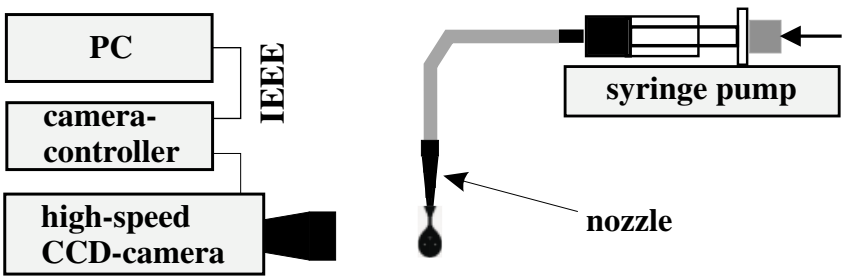

FIG. 1. A schematic diagram of the experimental setup. 
rates between 1000 and 6000 frames/s. An objective with a focal distance of $105 \mathrm{~mm}$ and an aperture of $37.5 \mathrm{~mm}$ diameter maps the drop onto the CCD with a magnification of 2.5. The frames are stored in the camera controller and analyzed by digital image processing. The fluid interface is detected as the maximum of the gradient of the image intensity, with an interpolation technique to optimize the spatial resolution. The fluid is technical glycerin with a water content of $16 \mathrm{wt} \%$. The material parameters amount to $\eta=99 \mathrm{mPas}, \sigma=64.6 \mathrm{mN} \mathrm{m}^{-1}$, and $\varrho=1.25 \mathrm{~g} \mathrm{~cm}^{-3}$, whereas the first two were measured with a rotational viscometer and a bubble pressure tensiometer. The values of the intrinsic length and time are $l_{\nu}=0.12 \mathrm{~mm}$ and $t_{\nu}=0.19 \mathrm{~ms}$.

Figure 2 shows a sequence of four pictures focusing at the immediate vicinity of the pinch point. The growing drop at the end of the pipette tip becomes unstable against gravity and begins to detach from the origin of the nozzle. The droplet appears fuzzy due to its downward movement. The coordinate system has been chosen according to the first picture in Fig. 2. In this frame of reference the point of pinch-off is situated at $z=0$ and the orifice of the nozzle is positioned at about $z=2 \mathrm{~mm}$.

In Fig. 3 we present the profiles of a drop as extracted from the original images obtained at a record rate of 6000 frames/s, with only every second line shown. The time proceeds from the outermost curve at the right-hand side to the one at the left-hand side. Drop formation is initiated by the thinning and elongation of the thread connecting the drop with the remaining fluid at the nozzle. The downward movement of the position $z_{\min }$ of the neck is accompanied by a transformation of the profile from a symmetric to an asymmetric one in a vicinity of $z_{\min }$.

The temporal evolution of the neck radius is expected $[5,9,3]$ to be described by a linear function

$$
h_{\min }=u_{(\mathrm{a}) \mathrm{s}} \sigma(\nu \varrho)^{-1}\left(t_{0}-t\right) .
$$

$u_{(\text {a)s }}$ denotes the predicted dimensionless shrink velocities. From Eq. (1) it can be seen that its value corresponds to the minimum of the scaling function $\Phi$, namely, $u_{\mathrm{s}}=0.071$ for the symmetric scaling function $\Phi_{\mathrm{s}}[9]$ and $u_{\mathrm{as}}=0.030$ for the asymmetric one $\Phi_{\mathrm{as}}[3,5]$, respectively.

To compare with those predictions, we determine the neck radius $h_{\min }$ by fitting the experimental data with a

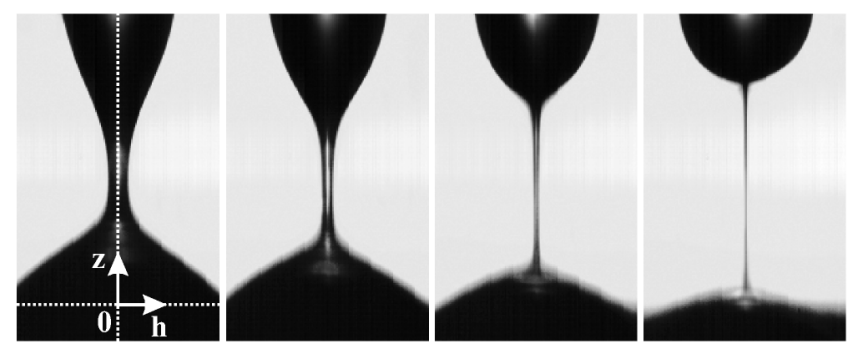

FIG. 2. The neck of a falling drop of a glycerin-water mixture before pinch-off. The time between consecutive images and the exposure time is $1 \mathrm{~ms}$. parabola in the immediate vicinity ( \pm 7 pixels corresponding to $175 \mu \mathrm{m}$ ) of their absolute minimum. The result of 20 measurements is presented in Fig. 4(a). The data show two different shrink velocities in two time intervals. Each interval can be fitted by a linear function. According to Eq. (2), the neck radius decreases first with the shrink velocity $v_{1}=45.3 \mathrm{~mm} / \mathrm{s}$ in good agreement with the predicted value $v_{\mathrm{s}}=46.3 \mathrm{~mm} / \mathrm{s}$ for a viscosity dominated flow. From the measured shrink velocity of the profile and the speed of the neck, we estimate a Reynolds number Re based on the maximal flow velocity in the vertical direction. The temporal evolution of that number is presented in Fig. 4(b). Because of the increase of Re, the theoretical assumption of negligible inertia appropriate for the early stages of the flow evolution is at a certain point no longer justified. At $t_{\mathrm{T}} \approx-2 \mathrm{~ms}$ and $\mathrm{Re} \approx 0.3 \mathrm{a}$ transition takes place between the two flow regimes. The neck radius shrinks now with $v_{2}=18.8 \mathrm{~mm} / \mathrm{s}$ until the border of our resolution is reached. Again the experimental value $v_{2}$ and the theoretical one $v_{\text {as }}=19.8 \mathrm{~mm} / \mathrm{s}$ are in good agreement.

We measure the asymmetry of the profiles with respect to the neck at $z_{\min }$ by

$$
\alpha=\left(\frac{\int h\left(z_{\min }+z^{\prime}\right)-h\left(z_{\min }-z^{\prime}\right) d z^{\prime}}{\int h\left(z_{\min }+z^{\prime}\right)+h\left(z_{\min }-z^{\prime}\right)-2 h_{\min } d z^{\prime}}\right)^{2} .
$$

$\alpha$ is zero for symmetric profiles and positive for asymmetric ones; it thus serves as an order parameter for the

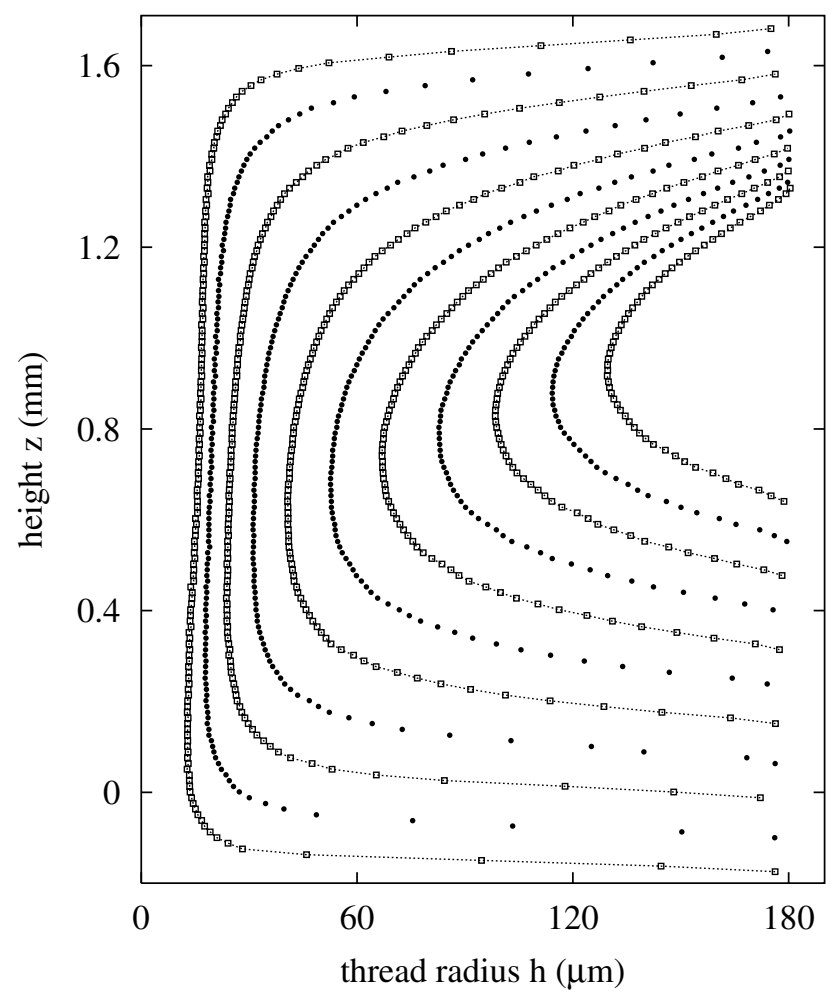

FIG. 3. The height $z$ and the thread radius $h$ as extracted from the original images. The outermost curve at the right-hand side (left-hand side) has been taken $3.83 \mathrm{~ms}(0.5 \mathrm{~ms})$ before the pinch-off. The dotted lines are a guide for the eye.

084501-2 


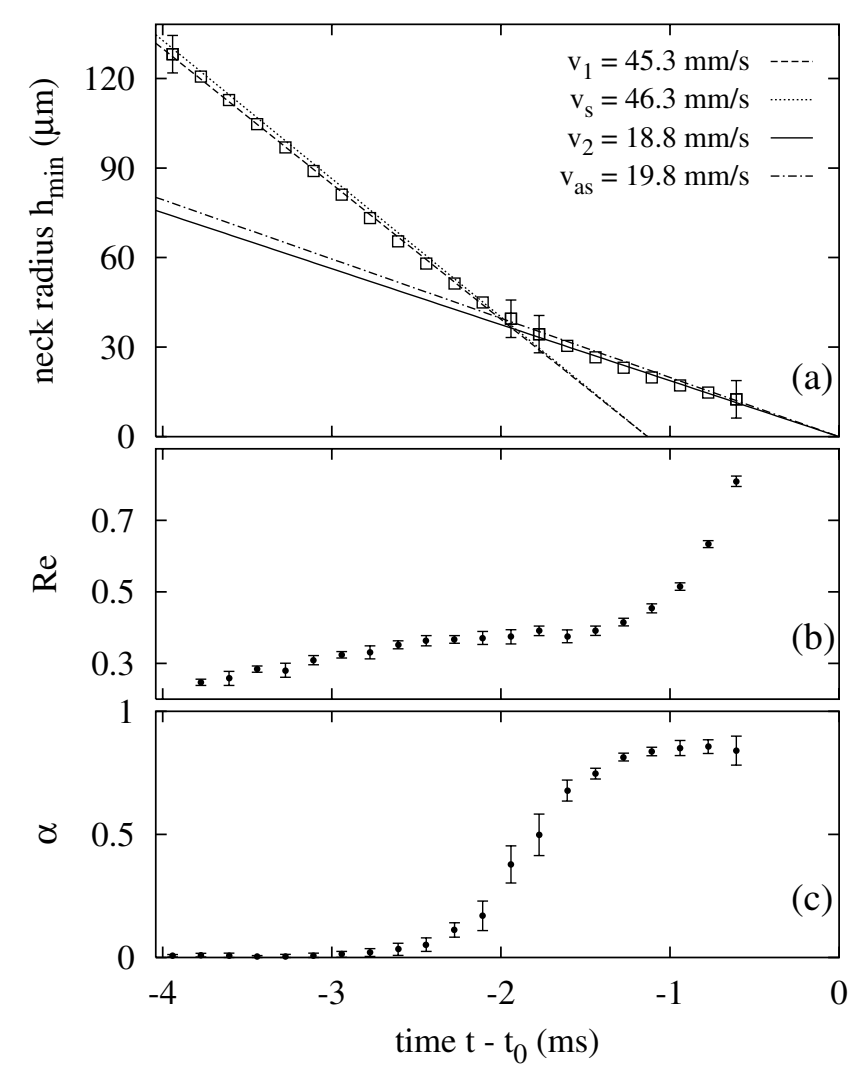

FIG. 4. The neck radius as extracted from parabolic fits to 20 experimental runs is marked by the open squares (a). The dotted line and the dashed line represent the theoretical prediction and a linear fit for the viscous-dominated flow regime [9]. The theoretical prediction and the linear fit for the Navier-Stokes flow [5] are marked by the dash-dotted line and the solid line, respectively. The error bars indicate the limited resolution due to the finite pixel size of the camera. $\operatorname{Re}$ in (b) represents the flow velocity in vertical direction. The asymmetry of the profiles is quantified in (c). The error bars in (b) and (c) indicate the standard deviation of the 20 measurements.

transition described here. The time dependence of $\alpha$ shown in Fig. 4(c) again indicates that this transition takes place around $t_{\mathrm{T}} \approx-2 \mathrm{~ms}$.

Once the different flow regimes have been discriminated, we check whether Eq. (1) is able to characterize the full profiles in the corresponding regimes by fitting to $h(z, t)=h_{\min } / \Phi_{\min } \Phi\left[\left(z-z_{\min }\right) a_{1}\right]$, where $\Phi$ represents $\Phi_{\mathrm{s}}$ or $\Phi_{\mathrm{as}}$, and $\Phi_{\min }$ the minimum of this scaling function. We use the time dependent position $z_{\min }$ of the neck radius as determined by the previous fit as a transformation to the frame comoving with the neck, thus taking into account the fluid motion caused by gravitation, which is not considered in the theory. Because of this force, the asymmetry has a well-defined direction in our experiment. The parameter $h_{\min }$ is determined by the previous fit as well. The remaining free parameter $a_{1}$ describes the stretching of the profile in time.

A priori it is not clear in which region in real space the scaling functions are valid. We restrict the area to a vicinity of the minimum where the slope is smaller than 0.5 , because the slender jet approximation is not expected to apply for large slopes. If the standard deviation is larger than an arbitrarily chosen value of $0.7 \mu \mathrm{m}$, those data points with the largest distance from the minimum are disregarded. The weighting factor for the fit decreases with increasing distance from the neck according to $1 / \sqrt{\left[\left(h / h_{\min }\right)-1\right]^{2}+\left[\left(z / z_{\min }\right)-1\right]^{2}+1}$. This two-stage algorithm (namely, to use a weighted fit after the minimum of the profile was determined independently) reflects the difficulty of fitting a function which is correct only locally, and turned out to be the most robust procedure.

The result of the fit procedure is presented in Fig. 5 for three representative profiles. The fit by the symmetric (asymmetric) scaling function is denoted by dashed (solid) lines. In contrast to Fig. 3 the plot is limited to the vicinity of the neck, where the fitting criteria apply. At the beginning of the observed time interval, at $t=-3.83 \mathrm{~ms}$ [Fig. 5(a)], a good agreement between the experimental data and $\Phi_{\mathrm{s}}$ can be found. At $t=-2.17 \mathrm{~ms}$ [Fig. 5(b)], the quality of the matching is about the same for the two theoretical predictions $\Phi_{\mathrm{s}}$ and $\Phi_{\mathrm{as}}$. Immediately before the pinch-off at $t=-0.5 \mathrm{~ms}$ [Fig. 5(c)], the measured profile is better described by $\Phi_{\mathrm{as}}$, the prediction for a Navier-Stokes flow. Thus during the pinch-off the drop profile changes from a symmetric to an asymmetric one,

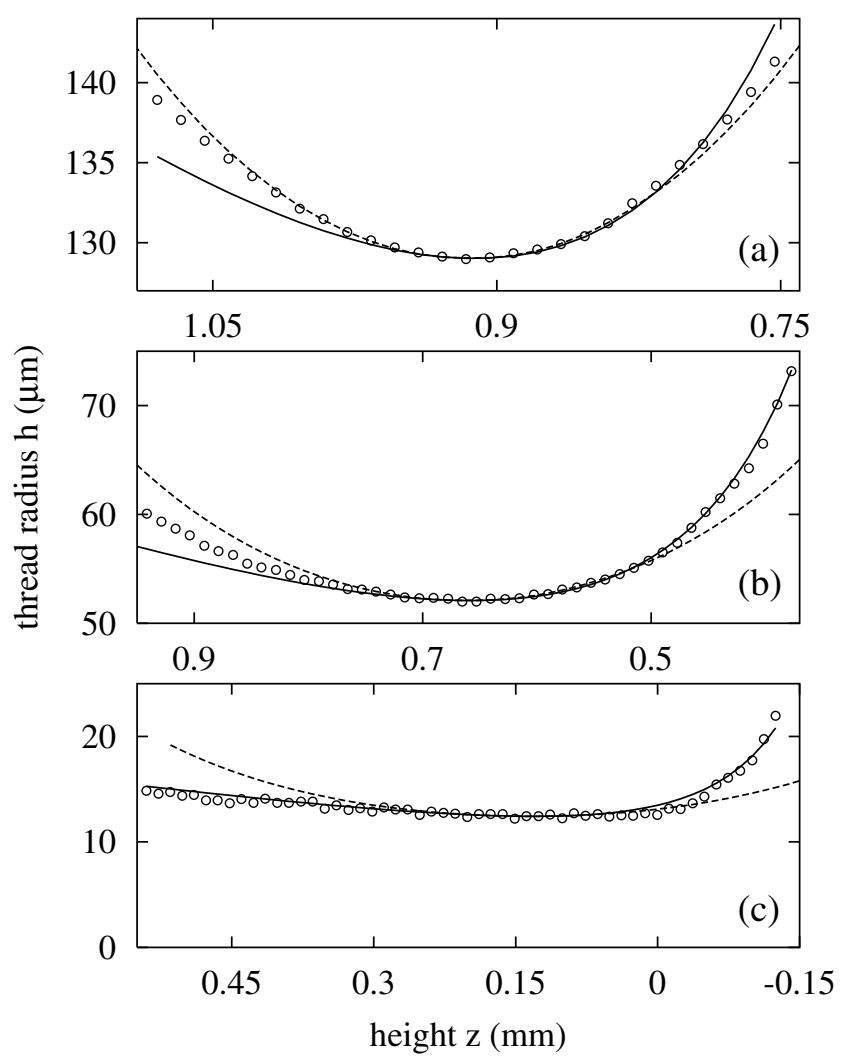

FIG. 5. The thread radius $h(z, t)$ at $t=-3.83 \mathrm{~ms}$ (a), $-2.17 \mathrm{~ms}$ (b), and $-0.5 \mathrm{~ms}$ (c) before the pinch-off. The fitted $\Phi_{\mathrm{s}}\left(\Phi_{\mathrm{as}}\right)$ is indicated by the dashed (solid) line. 


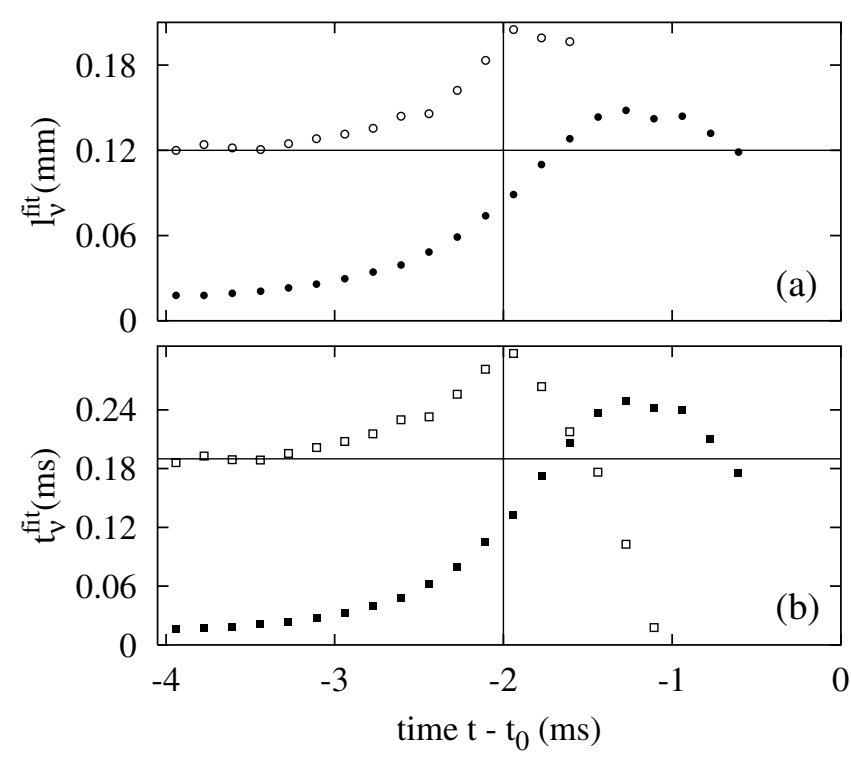

FIG. 6. The intrinsic length (a) and time (b) as extracted from the fit parameters of Eq. (1). The calculated values according to $\Phi_{\mathrm{s}}\left(\Phi_{\mathrm{as}}\right)$ are shown as open (solid) symbols, respectively. The horizontal lines mark the measured values, and the vertical ones the transition point $t_{\mathrm{T}}$.

which indicates the transition from the viscous-dominated to the inertia-dominated flow.

To check the theoretical predictions for the temporal evolution of the profiles, we investigate the development of the fit parameters in time. More precisely, from the fit to $\Phi_{\text {as }}$ we extract $l_{\nu}^{\text {fit }}=1 /\left(a_{0} a_{1}^{2}\right)$ and $t_{\nu}^{\mathrm{fit}}=\left(t_{0}-t\right) /\left(a_{0} a_{1}\right)^{2}$ with $a_{0}=h_{\min } / \Phi_{\min }$. The corresponding values are shown in Fig. 6 as solid symbols. In the Stokes-flow regime, i.e., before the vertical line which marks the transition point $t_{\mathrm{T}}$, the values are considerably smaller than the ones determined independently from the measured fluid parameters (horizontal lines). They increase and have a flat maximum in the regime of the Navier-Stokes flow, where $\Phi_{\mathrm{as}}$ applies. $l_{\nu}^{\mathrm{fit}}$ and $t_{\nu}^{\mathrm{fit}}$ are expected to be constant within the framework of the theory. The deviation from the measured value $l_{\nu}^{\text {meas }}$ and $t_{\nu}^{\text {meas }}$ before the transition point is explained by the fact that $\Phi_{\mathrm{as}}$ is not the appropriate scaling function in this regime. After this transition an agreement within $20 \%$ can be observed. The results from a fit to the symmetric function are also indicated in Fig. 6 . In contrast to $\Phi_{\text {as }}$ the width of $\Phi_{\mathrm{s}}$ can be derived only up to an arbitrary normalization length $\bar{\xi}$, which is determined by the initial condition of the experiment $[9,3]$. We thus determine $\bar{\xi}=a_{1}(t) l_{\nu}^{\text {meas }}\left[\left(t_{0}-t\right) / t_{\nu}^{\text {meas }}\right]^{\beta}$ by taking the mean value within the first $0.8 \mathrm{~ms}$ of the time interval shown in Fig. 6. With that value of $\bar{\xi}$, the intrinsic length and time can be extracted by $l_{\nu}^{\text {fit }}=a_{0}\left(a_{0} a_{1} \bar{\xi}^{-1}\right)^{1 /(\beta-1)}$, and $t_{\nu}^{\mathrm{fit}}=\left(t_{0}-t\right)\left(a_{0} a_{1} \bar{\xi}^{-1}\right)^{1 /(\beta-1)}$ with the scaling exponent $\beta=0.175$. The results are shown as open symbols in Fig. 6. During the longer part of the Stokes-flow regime $l_{\nu}^{\mathrm{fit}}$ is constant; it increases at the transition point $t_{\mathrm{T}}$. After $t_{\mathrm{T}}$ the values scatter. In fact, some of the values do not even fall in the range of the plot. This is explained by the fact that a description by $\Phi_{\mathrm{s}}$ is not appropriate in the final state before the pinch-off.

In conclusion, we have experimentally confirmed that sufficiently far from the pinch-off the shrinking neck of a glycerin-water mixture is well described by a Stokes-flow description [9]. This refers both to the shape and the shrink velocity of the neck radius. Immediately before the pinch-off, a different shrink velocity and geometrical shape as obtained for the case of a Navier-Stokes flow [5] could also be corroborated. The transition from one flow form to the other takes place at $t_{\mathrm{T}} \approx 10 t_{\nu}$ and $\mathrm{Re} \approx 0.3$.

Further measurements will have to unveil the viscosity dependence of $t_{\mathrm{T}}$. Moreover, it seems important to replace the surrounding air used in our experiment by other fluids [10], because Lister and Stone [11] suggest a further transition from Navier-Stokes flow to a two fluid Stokes flow in this case. That transition has not yet been resolved experimentally.

The authors thank N. Aksel, Th. M. Bock, and L. Heymann for valuable discussions and technical support, J. Eggers for putting the scaling functions to our disposal, and "Deutsche Forschungsgemeinschaft" (Grant No. Re588/12) for financial support.

[1] M. Mariotte, Traite du Mouvement des Aux et des Autres Corps Fluides (Claude-Jombert, Paris, 1718), 2nd ed.

[2] J. W. S. Rayleigh, Philos. Mag. 34, 145 (1892).

[3] J. Eggers, Rev. Mod. Phys. 69, 865 (1997).

[4] H. C. Lee, IBM J. Res. Dev. 18, 364 (1974).

[5] J. Eggers, Phys. Rev. Lett. 71, 3458 (1993).

[6] T. A. Kowalewski, Fluid Dyn. Res. 17, 121 (1996).

[7] X. D. Shi, M. P. Brenner, and S. R. Nagel, Science 265, 219 (1994).

[8] M. P. Brenner, J. R. Lister, and H. A. Stone, Phys. Fluids 8, 2827 (1996).

[9] D. T. Papageorgiou, Phys. Fluids 7, 1529 (1995).

[10] I. Cohen, M. P. Brenner, J. Eggers, and S. R. Nagel, Phys. Rev. Lett. 83, 1147 (1999).

[11] J. R. Lister and H. A. Stone, Phys. Fluids 10, 2758 (1998). 